\title{
Diffuse reflectance spectroscopy biomarkers for biological tissues characterization: application to ex- vivo animal tissues
}

F. Fanjul-Vélez, J. L. Arce-Diego

F. Fanjul-Vélez, J. L. Arce-Diego, "Diffuse reflectance spectroscopy biomarkers for biological tissues characterization: application to ex-vivo animal tissues," Proc. SPIE 10873, Optical Biopsy XVII: Toward Real-Time Spectroscopic Imaging and Diagnosis, 108730N (4 March 2019); doi: $10.1117 / 12.2508954$

SPIE. Event: SPIE BiOS, 2019, San Francisco, California, United States 


\title{
Diffuse Reflectance Spectroscopy biomarkers for biological tissues characterization: application to ex-vivo animal tissues
}

\author{
F. Fanjul-Vélez*, J. L. Arce-Diego* \\ Applied Optical Techniques Group, TEISA Department, University of Cantabria, Av de los Castros \\ s/n, 39005, Santander (Spain)
}

\begin{abstract}
Biological tissues characterization can be approached by non-ionizing optical techniques, in a non-invasive, non-contact way. Optical diagnostic techniques include Optical Coherence Tomography, spectroscopy or fluorescence, among others. Tissue differentiation is difficult to achieve in general with high specificity and sensibility. Spectroscopy is of great interest for this aim, as it provides intrinsic molecular contrast. The different composition and/or structure of biological tissues influence the spectral response. However, the interpretation of spectra is difficult from the raw data, and further data processing is needed. Diffuse Reflectance Spectroscopy (DRS) is particularly well-suited for biomedical applications, as it can work with bulk tissues in reflection, reinforcing the non-invasive character of the technique. DRS has been employed for malignant tissue detection and also for healthy tissue discrimination. These applications require an adequate definition of potential biomarkers for the classification algorithms. The classification process depends strongly on the amount of collected spectra and tissue and specimen variability. In this work several types of ex-vivo porcine tissues are extracted and measured by DRS. Spectral measurements are made on different specimens, and on different points of each sample. Spectra are normalized and several algorithms for dimension and variability reduction are applied, such as Principal Component Analysis or Savitzky-Golay filtering. From these spectra, several biomarkers are proposed for tissue classification, and different classifiers are applied. The results are compared, and the classification efficiency is quantified. The considered approaches could be of particular interest in image-guided surgery or other types of optical biopsy applications.
\end{abstract}

Keywords: Diffuse Reflectance Spectroscopy, biological tissue discrimination, tissue diagnostics, tissue classification

\section{INTRODUCTION}

Biological tissues characterization can be approached by non-ionizing optical techniques, in a non-invasive, non-contact way. Optical characterization can be implemented by different optical diagnostic techniques, such as Optical Coherence Tomography (OCT) [1] and its variants [2], spectroscopy [3], fluorescence [4], or even polarimetry [5]. Spectroscopy provides in general tissue-specific contrast at molecular level. Diffuse Reflectance Spectroscopy (DRS) is particularly well-suited for biomedical applications, as it can work with bulk tissues in reflection, reinforcing the non-invasive character of the technique. [6]. DRS is an affordable technique capable of providing data on tissue morphology and/or functionality. DRS has been applied mainly for the detection of cancerous tissues [4], but can be clinically applied in general classification problems, for instance in the diagnosis of pathologies. In this way, each sample is classified as healthy or diseased tissue. Tissue type discrimination can be further implemented [7]. Statistically significant classifications are supported by massive spectral measurements of each class. Those measurements come from different samples of the same specimen, and from different specimens. This fact leads to spectral variability, whose relevance must be estimated [6]. In this work several types of ex-vivo porcine tissues are extracted and measured by DRS. Spectral measurements are made on different specimens, and at different points of each sample. Spectra are normalized and several algorithms for dimension and variability reduction are applied, such as Principal Component Analysis or Savitzky-Golay filtering. From these spectra, several biomarkers are proposed for tissue classification, and different classifiers are applied. The results are compared, and the classification efficiency is quantified. Next section 2 contains the information on animal samples, optical setup and spectral measurements. Main algorithms employed are also included. Section 3 shows some of the results obtained. Finally section 4 includes the conclusions of the work.

*fanjulf@unican.es; arcedj@unican.es; phone +34 94220 67 30; fax +34 9422018 73; www.teisa.unican.es/toa

Optical Biopsy XVII: Toward Real-Time Spectroscopic Imaging and Diagnosis, edited by

Robert R. Alfano, Stavros G. Demos, Angela B. Seddon, Proc. of SPIE Vol. 10873

$108730 \mathrm{~N} \cdot$ ? 2019 SPIE $\cdot$ CCC code: $1605-7422 / 19 / \$ 18 \cdot$ doi: $10.1117 / 12.2508954$ 


\section{MATERIALS AND METHODS}

Pigs of the Large White breed were used for the experiments. The animals were between 2 and 4 months old and their weight between 20 and $25 \mathrm{~kg}$. Five types of ex vivo tissues were extracted from pigs, particularly skin, fat, muscle, bone (rib) and sciatic nerve. Two samples of the same tissue from each pig were taken. The procedure was done at the hospital by a veterinary surgeon, who handles carefully the tissue without causing any mechanical damage. The procedure was approved by the Bioethical Committee of the Valdecilla Virtual Hospital. Fig. 1 shows the tissue samples placed on purposely designed holders to fit into the experimental setup. A tissue surface of $30 \times 30 \mathrm{~mm}^{2}$ is accessible to be measured, with the exception of bones and nerves, which have a mean diameter of $20 \mathrm{~mm}$ and $1 \mathrm{~mm}$, respectively. After dissection, tissues were carefully rinsed with a solution of sodium chloride $(9 \mathrm{mg} / \mathrm{ml})$ and wrapped with soaked gauze. The samples were stored in the refrigerator $\left(4^{\circ}\right)$, where they remain up to a maximum of 12 hours until the samples are processed.
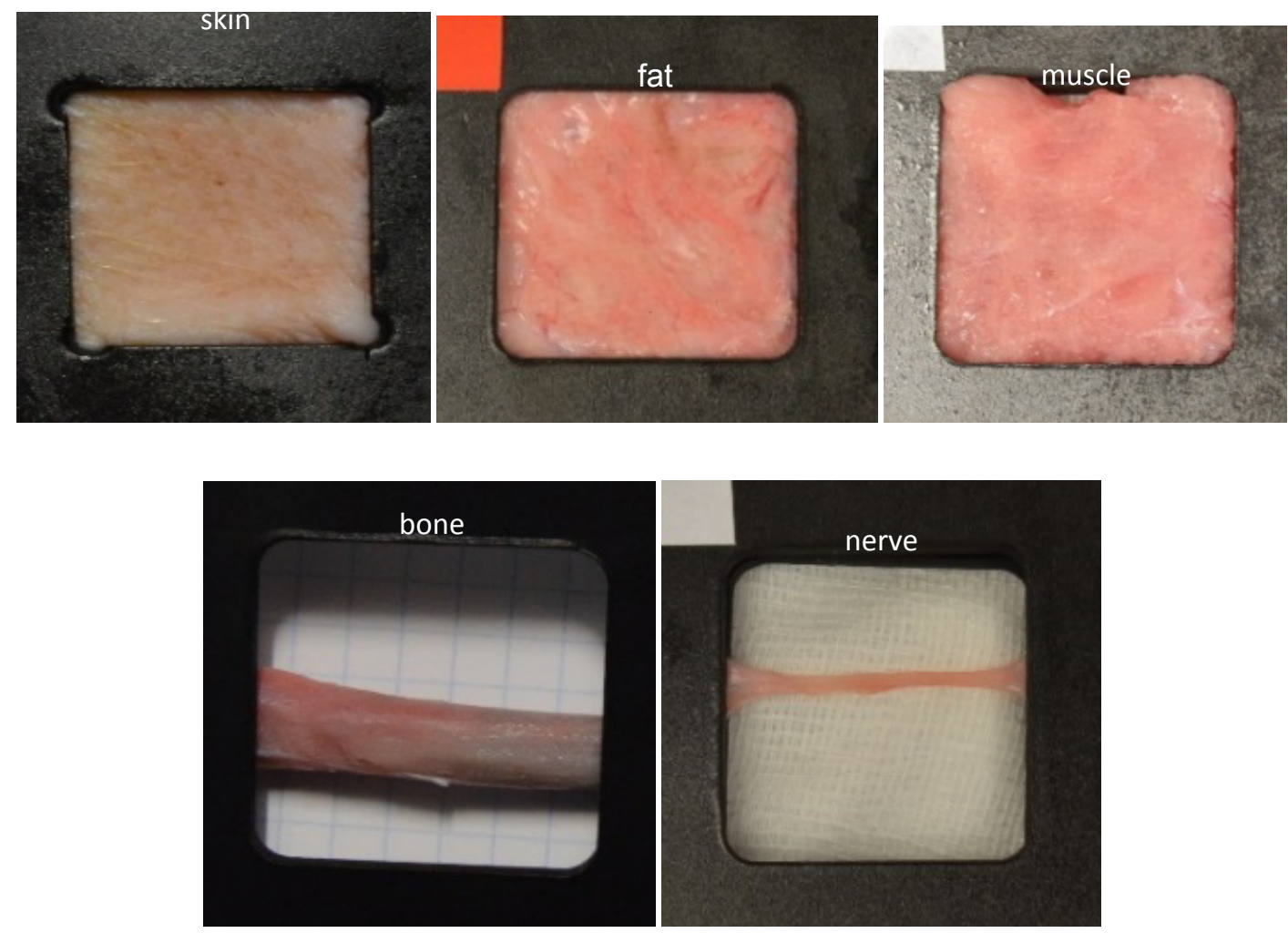

Figure 1. Tissue samples from ex vivo pigs.

In Fig. 2, the experimental setup is sketched. The system employs a white light source $250 \mathrm{~W}$ broadband incandescent lamp (QTH 66499, Newport Corporation). The lamp exhibits a smooth spectral curve in a wavelength range of 200-2400 $\mathrm{nm}$. The tissue was illuminated with the light through a couple of lenses made of fused silica $\left(\mathrm{L}_{1}\right.$ and $\left.\mathrm{L}_{2}\right)$. The diffusely reflected light from the tissue is collected by a lens, and focused into a $1 \mathrm{~mm}$ core diameter optical fiber, which is connected to a spectrometer (BLK-CXR-SR-50, StellarNet Inc.), with a measurement range between 220-1100nm, an optical resolution of $0.5 \mathrm{~nm}$, an integration time of $50 \mathrm{~ms}$, and a 2048-pixel CCD/PDA detector with a size of $14 \times 200$ $\mu \mathrm{m} /$ pixel. The observation was made obliquely at $30^{\circ}$.

For each tissue from each animal 16 spots were scanned by using a micrometric translator. Spots were spread out on a $4 \times 4$ matrix, so each point was separated approximately $6 \mathrm{~mm}$ from each other. Four spectra were captured per spot, and as a consequence a total of 1280 spectra were processed in this work. 


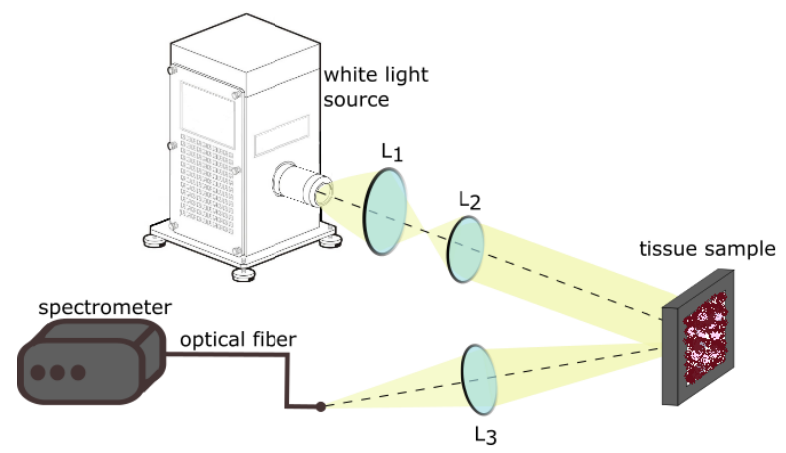

Fig. 2. Experimental setup of Diffuse Reflectance Spectroscopy.

The reflectance spectrum of the tissue $R_{\text {tissue }}(\lambda)$ is found from the captured signal $S_{\text {tissue }}(\lambda)$ and the light source emission spectrum $S_{\text {light }}(\lambda)$, which is obtained with a reflectance standard (WS-1 Ocean Optics, 250-1500 nm). The background signal $S_{\text {background }}(\lambda)$ is subtracted from the two signals, and its ratio is calculated:

$$
R_{\text {tissue }}(\lambda)=\frac{S_{\text {tissue }}(\lambda)-S_{\text {background }}(\lambda)}{S_{\text {light }}(\lambda)-S_{\text {background }}(\lambda)} .
$$

A pre-treatment is needed in order to remove systematic noise undesirable variations (due to the measuring instrument and the sample itself) and emphasize the variations of interest. A detrend analysis removes linear trends and a SavitzkyGolay filter removes noise. The spectra are normalized by subtracting its mean and dividing by its standard deviation [8]. Principal Component Analysis (PCA) is employed to reduce dimensionality. PCA is a well-known technique to reduce the dimensionality of the problem. The algorithm transforms a number of possibly correlated variables into a number of equal or smaller, uncorrelated (orthogonal) variables, which are named as principal components (PC). The algorithm projects the data on a space of lower dimension while the variance of the projected data is maximized. Each spectrum has 1749 data points, since the signal was captured in a range of $226 \mathrm{~nm}$ to $1100.5 \mathrm{~nm}$, every $0.5 \mathrm{~nm}$. Each spectrum represents a point in $\mathbb{R}^{1749}$. The distance between each observation and the centroid of its class is measured by the Mahalanobis distance.

\section{RESULTS AND DISCUSSION}

The previously reported setup is employed to obtain the measurements, and the spectra are analyzed according to the algorithms exposed. In the PCA space each tissue is analyzed separately. In order to obtain further information, observations coming from the same specimen are drawn with the same color. Fig. 3 shows an example with the fat and the muscle tissues. Two samples (A and B) of two specimens (1 and 2) are shown. It is clear by observing the graphs that each sub-class is bound together forming clusters, so a discrimination can be made.
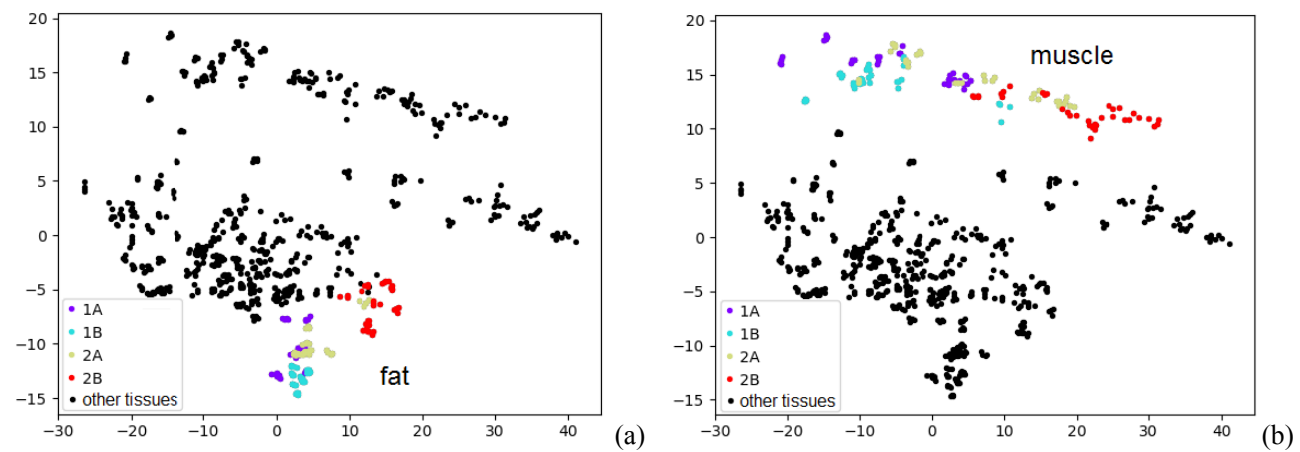

Fig. 3. PC space where a type of tissue has been highlighted in colours (black points correspond to the rest of tissues). (a) Fat class. (b) Muscle class. Different colours correspond to different specimens and/or samples. 
According to the Mahalanobis distance, a classification area can be defined for each tissue type by means of an ellipse. As an example, Fig. 4 shows classifications ellipses of fat tissue, first with all the samples considered (Fig. 4a)), and afterwards when any of the samples is suppressed (Fig. 4b) to 4e)). The influence of the particular samples that are included in the analysis is clear from the graphs.

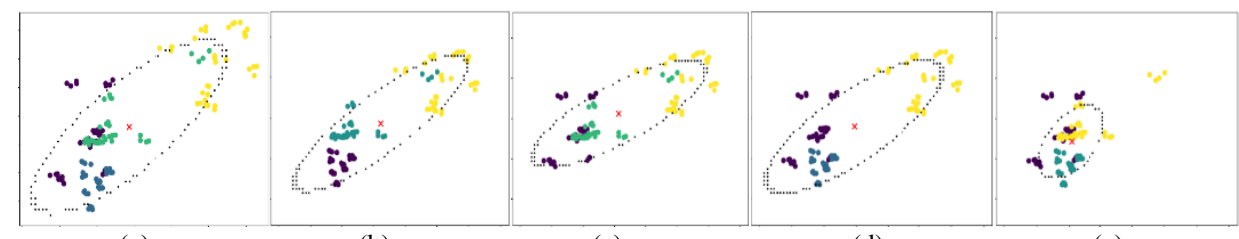

(a)

(b)

(c)

(d)

(e)

Fig. 4. Classification ellipses based on PC components for fat tissue. (a) Al the samples are included. (b)-(e) When data coming from one specimen is removed, the area that characterizes each class changes.

\section{CONCLUSIONS}

DRS as a classification technique is a promising approach. Several optical reflectance spectra of ex-vivo porcine tissues from different samples and animals have been measured. Usual normalization procedures have been applied. Variability between data coming from different samples and animals has been found, evidenced in the PCA space, and measured by using Mahalanobis distance. This analysis can be relevant for the classification and discrimination problems in biological tissues employing DRS, such as image guided surgery.

\section{ACKNOWLEDGEMENTS}

This work has been partially supported by the project "New active phases in transition metals and rare earth nano-oxides stabilized at high pressure" (MAT2015-69508-P) of the Spanish Ministry of Economy and Competitiveness, cofunded by FEDER funds, and by the San Cándido Foundation.

\section{REFERENCES}

[1] Brezinski, M. E., Tearney, G. J., Bouma, B., Boppart, S. A., Pitris, C., Southern, J. F. and Fujimoto, J. G., "Optical biopsy with optical coherence tomography," Ann. Ny. Acad. Sci. 838(1), $68-74$ (1998).

[2] Fanjul-Vélez, F., Pircher, M., Baumann, B., Götzinger, E., Hitzenberger, C. K. and Arce-Diego, J. L., "Polarimetric analysis of the human cornea by Polarization Sensitive Optical Coherence Tomography," J. Biomed. Opt. 15, 056004 (2010).

[3] García-Uribe, A., Kehtarnavaz, N., Marquez, G., Prieto, V., Duvic, M. and Wang, L. V., "Skin cancer detection by spectroscopic oblique-incidence reflectometry: classification and physiological origins," Appl. Optics 43(13), 2643-2650 (2004).

[4] Salas-García, I., Fanjul-Vélez, F. and Arce-Diego, J. L., "Superficial radially-resolved fluorescence and threedimensional photochemical time-dependent model for Photodynamic Therapy," Opt. Lett. 39, 1845-1848 (2014).

[5] Ortega-Quijano, N., Fanjul-Vélez, F., de Cos-Pérez, J. and Arce-Diego, J. L., "Analysis of the depolarizing properties of normal and adenomatous polyps in colon mucosa for the early diagnosis of precancerous lesions," Opt. Commun. 284, 4852-4856 (2011).

[6] Fanjul-Vélez, F., Arévalo-Díaz, L. and Arce-Diego, J. L., "Intra-class variability in diffuse reflectance spectroscopy: application to porcine adipose tissue," Biomedical Optics Express 9, 2297-2303 (2018). 
[7] Stelzle, F., Tangermann Gerk, K., Adler, W., Zam, A., Schmidt, M., Douplik, A. and Nkenke, E., "Diffuse reflectance spectroscopy for optical soft tissue differentiation as remote feedback control for tissue specific laser surgery," Laser Surg. Med. 42(4), 319-325 (2010).

[8] Barnes, R. J., Dhanoa, M. S. and Lister, S. J., "Standard normal variate transformation and de-trending of nearinfrared diffuse reflectance spectra," Appl. Spectrosc. 43(5), 772-777 (1989). 